\title{
First checklist of rust fungi in the genus Puccinia from Himachal Pradesh, India
}

\section{Gautam $\mathrm{AK}^{1^{*}}$ and Avasthi $\mathrm{S}^{2}$}

\author{
${ }^{1}$ Faculty of Agriculture, Abhilashi University, Mandi-175028, India \\ ${ }^{2}$ Department of Botany, Abhilashi Institute of Life Sciences, Mandi- 175008, India
}

Gautam AK, Avasthi S 2016 - First checklist of rust fungi in the genus Puccinia from Himachal Pradesh, India. Plant Pathology \& Quarantine 6(2), 106-120, Doi 10.5943/ppq/6/2/1

\begin{abstract}
A checklist of rust fungi belonging to the genus Puccinia was prepared for Himachal Pradesh, India. All Puccinia species published until 2014 are included in this list. A total of 80 species have been reported on 91 plant species belonging to 33 families. The family Poaceae supports the highest number of species (26 species) followed by Ranunculaceae (8), Asteraceae (7), Apiaceae and Polygonaceae (6 each), Rubiaceae and Cyperaceae (3 each), Acanthaceae, Berberidaceae, Lamiaceae and Saxifragaceae (2 each). The other host plant families are associated with a single species of Puccinia. This study provides the first checklist of Puccinia from Himachal Pradesh.
\end{abstract}

Key words - checklist - Himachal Pradesh - Puccinia spp. - rust fungi

\section{Introduction}

Himachal Pradesh is a hilly state situated in the heart of Himalaya in the northern part of India. The state extends between $30^{\circ} 22^{\prime} 40^{\prime \prime}-33^{\circ} 12^{\prime} 20^{\prime \prime}$ north latitudes and $75^{\circ} 44^{\prime} 55^{\prime \prime}-79^{\circ}$ $04^{\prime} 20^{\prime}$ " east longitudes. The total area of the state is $55,670 \mathrm{~km}^{2}$, covered with very high mountains to plain grasslands. It is a mountainous state with elevation ranging from about 350 to 7,000 meters above sea level. The climatic conditions vary from hot and sub-humid tropical in the southern tracts to cold, alpine and glacial in the northern and eastern mountain ranges with increasing elevation. These variations lead to great biodiversity in the state. Two-thirds of the area is covered by very dense evergreen to deciduous forest types. Alpine shrub and meadows are found in west and northeast Himalaya; alders, birches, rhododendrons and moist alpine shrubs are found as the regional vegetation. Himachal Pradesh has 12 major national parks and sanctuaries to conserve the flora and fauna of the main Himalayan range. However, the main threat for extinction of floral diversity is no doubt, the activities of human beings, but plant diseases also contribute to decline. The changeable geographical and climatic conditions of the state are favourable for the growth and development of plant pathogens. Bacteria, fungi and viruses have been reported as plant pathogens from the state of which rusts contribute as the main fungal group reported here. Much is known about these fungi from the state. One of the most important characteristics of plant rusts is their exceptionally high degree of host specificity. The vast biodiversity and climatic conditions of the 
state contribute to the vast diversity, distribution and host range of this fungal group (http://hpplanning.nic.in; https://en.wikipedia.org/wiki/Geography_of_Himachal_Pradesh).

Puccinia Pers. (1801) is an obligate plant pathogen genus and contains about 4,000 species. This checklist provides the first comprehensive overview of the rust genus Puccinia in Himachal Pradesh. It facilitates access to scattered Himalayan literature with special reference to the state on the topic that may not be readily available to students studying plant pathology in India and abroad.

\section{Materials \& Methods}

This checklist was based on an exhaustive bibliographic survey of the literature published in various national and international journals, monographs, books, book chapters and even magazines on the rust genus Puccinia. A geographical map of the state is provided in order to understand the distribution of the rust fungi in Himachal Pradesh (Fig. 1). Family wise list of susceptible plant host was prepared to assess the host range of Puccinia in the state. Species distribution was assessed on the basis of their occurrence on plant host and their families and their geographical distribution. A brief description on taxonomy and occurrence of this fungus in different regions of the country is also presented. Some species names as reported in the cited publications have been replaced with their currently accepted name according to the Species Fungorum (www.speciesfungorum.org) website.

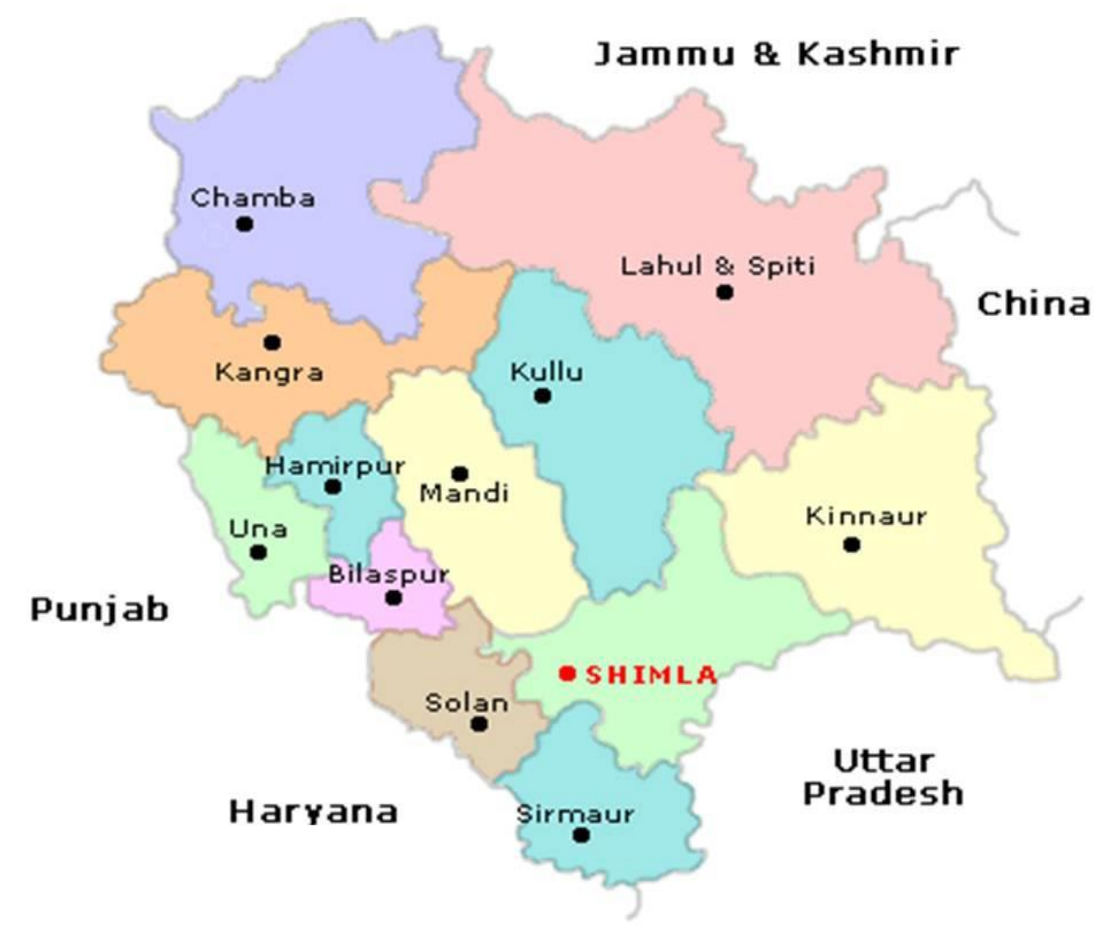

Fig 1 - Map of Himachal Pradesh

\section{Results}

A total of 80 species of Puccinia have been identified and reported from Himachal Pradesh. The rusts were recorded on 91 plant host species that belong to 80 genera and 33 families. Twentysix species were found associated with family Poaceae followed by Ranunculaceae (8), Asteraceae (7), Apiaceae and Polygonaceae (6 each), Rubiaceae and Cyperaceae (3 each), Acanthaceae, Berberidaceae, Lamiaceae, and Saxifragaceae ( 2 each). The remaining host plant families were associated with a single species of Puccinia (Table 1). After compilation of the literature it was noticed that most of the rust fungi were reported from Shimla and nearby regions. However, reports are also available from various regions of Solan, Kangra, Kullu, Chamba, Hamirpur, Una and even 
Lahul Spiti. In addition to Himachal Pradesh, the fungal group was found distributed outside the state as from Jammu \& Kashmir (J\&K); Punjab (PB); Haryana (HR); Delhi; Uttrakhand (Uttk); Uttar Pradesh (UP); Rajasthan (RJ); Madhya Pradesh (MP); Gujarat (GJ); Bihar, Chhattisgarh, Tamil Nadu (TN); Andhra Pradesh (AP); Maharashtra (MS); Karnataka; Kerala; Assam; West Bengal (WB) and Nagaland (NL). The abbreviated state names are used in the list. The morphological appearance of the rust pathogen on plant hosts and microscopic characteristics of urediniospores and teliospores are presented (Figs 2, 3).

Table 1 Puccinia species reported from Himachal Pradesh on different host families.

\begin{tabular}{|c|c|c|}
\hline Family & Puccinia species & $\begin{array}{c}\text { Number of } \\
\text { Puccinia species }\end{array}$ \\
\hline Acanthaceae & P. tweediana, $P$. polliniae & 2 \\
\hline Amaryllidaceae & P. allii & 1 \\
\hline Apiaceae & $\begin{array}{l}\text { P. bupleuri-falcate, } P \text {. ellisii, } P \text {. pimpinellae, } P \text {. apii, } \\
\text { P. bulbocastani, } P \text {. saviculae }\end{array}$ & 6 \\
\hline Asteraceae & $\begin{array}{l}P . \text { ahmadiana, } P \text {. atropuncta, } P \text {. carthami, } P \text {. sonchi, } \\
P . \text { tanaceti, } P \text {. tricholepidis, } P . \text { opizii }\end{array}$ & 7 \\
\hline Balsaminaceae & P. komarovi & 1 \\
\hline Berberidaceae & P. brachypodii, $P$. calthae & 2 \\
\hline Brassicaceae & P. thlaspeos & 1 \\
\hline Caryophyllaceae & P. arenariae & 1 \\
\hline Cyperaceae & $\begin{array}{l}P . \text { caricis var. himalayensis, } P \text {. caricis-filicinae, } P \text {. } \\
\text { caricis-nubigenae }\end{array}$ & 3 \\
\hline Discoraceae & P. dioscoreae & 1 \\
\hline Gentianaceae & P. gentianae & 1 \\
\hline Geraniaceae & P. geranii-sylvaticae & 1 \\
\hline Iridaceae & P. iridis & 1 \\
\hline Lamiaceae & P. excels, P. menthae & 2 \\
\hline Oleaceae & P. chrysopogi & 1 \\
\hline Onagraceae & P. circaeae & 1 \\
\hline Oxalidaceae & P. sorghi & 1 \\
\hline Plantaginaceae & P. pacifica & 1 \\
\hline Poaceae & $\begin{array}{l}P . \text { andropogonis, } P . \text { anthistiriae, } P . \text { arthraxonis, } P . \\
\text { chrysopogi, } P . \text { coronata, } P . \text { coursiniae, } P . \\
\text { cynodontis, } P . \text { dactylidina, } P . \text { duthiae, } P . \text { eulaliae, } P \text {. } \\
\text { erianthi, } P . \text { glumarum, } P . \text { graminis, } P \text {. graminis- } \\
\text { agropyri, } P \text {. graminis-poae, } P \text {. himalensis, } P . \\
\text { invenusta, } P . \text { neyaudiae, } P \text {. minutissima, } P . \\
\text { pogonatheri, } P \text {. purpurea, } P . \text { pusilla, } P \text {. recondite } \\
\text { var. simlensis, } P \text {. sorghi, } P \text {. striiformis, } P . \text { striiformis } \\
\text { f. muehlenbergii }\end{array}$ & 26 \\
\hline Polygonaceae & $\begin{array}{l}P . \text { bistortae, } P . \text { fagopyri, } P . \text { nepalensis, } P . \text { nitida, } P \text {. } \\
\text { polygone, } P \text {. polygone-amphibii }\end{array}$ & 6 \\
\hline Ranunculaceae & $\begin{array}{l}P . \text { agrostidis, } P \text {. calthae, } P \text {. lycoctoni, } P \text {. persistens, } \\
P \text {. recondita var. simlensis, } P \text {. rubigo-vera, } P \text {. trolii, } \\
P \text {. ustalis }\end{array}$ & 8 \\
\hline Rhamnaceae & P. himalensis & 1 \\
\hline
\end{tabular}




\section{Table 1 continued}

\begin{tabular}{llc}
\hline \multicolumn{1}{c}{ Family } & \multicolumn{1}{c}{ Puccinia species } & $\begin{array}{c}\text { Number of } \\
\text { Puccinia } \text { species }\end{array}$ \\
\hline Rosaceae & P. flavipes & 1 \\
Rubiaceae & P. colletiana, P. leptodermidis, P. puctata & 3 \\
Saxifragaceae & P. saxifragae-cilliatae, P. saxifragae-micranthae & 2 \\
Smilacaceae & P. prainiana & 1 \\
Urticaceae & P. urticae & 1 \\
Violaceae & P. violae & 1 \\
Xanthorrhoeaceae & P. eremuri & 1 \\
Zingiberaceae & P. roscoeae & 1 \\
\hline
\end{tabular}
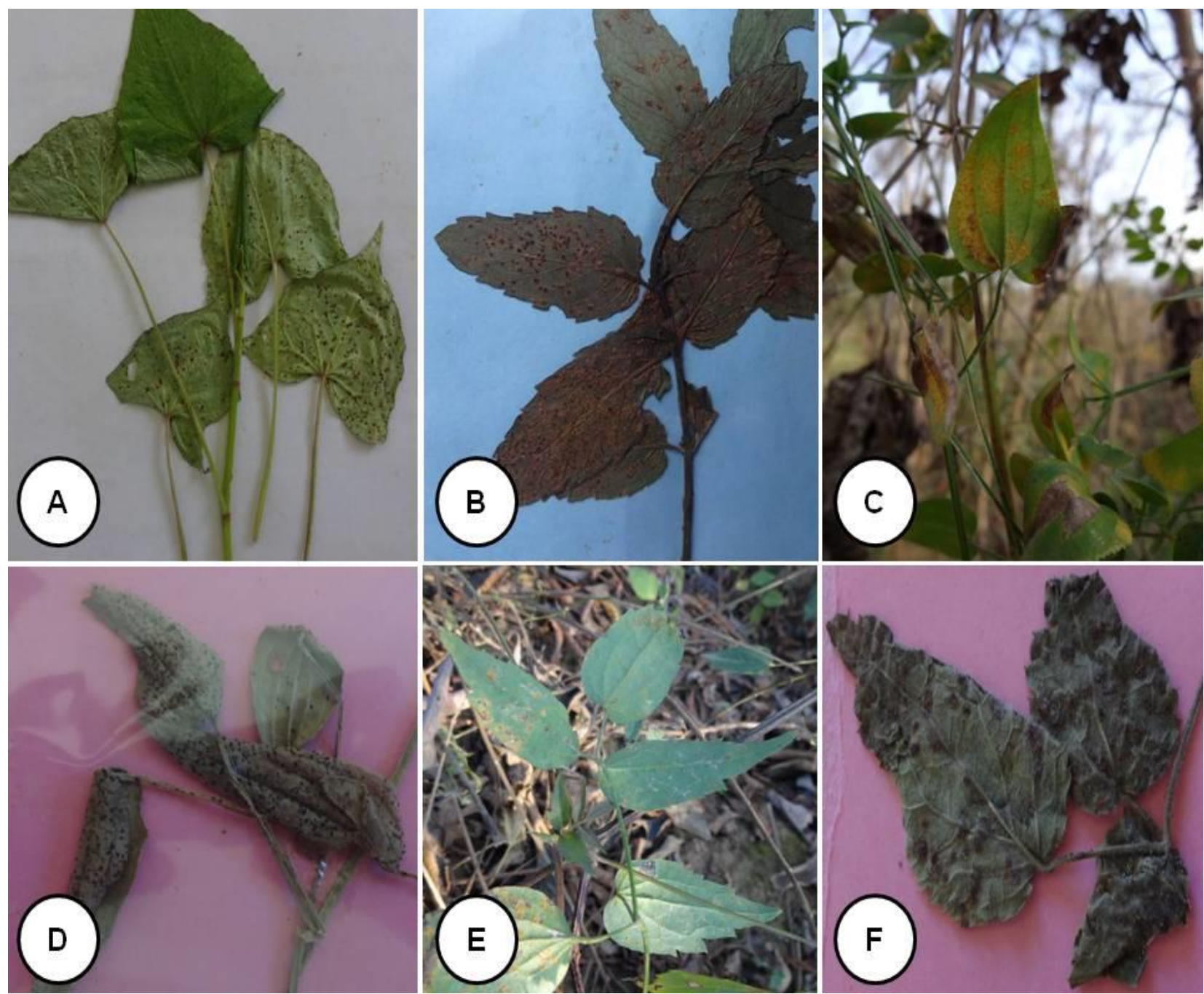

Fig 2 - Plant hosts showing rust infection. A) Puccinia fagopyri on Fagopyrum esculentum B) P. menthae on Mentha longifolia C, D P. colletiana on Rubia cordifolia (uredinia and telia) E, F Puccinia sp. on Clematis sp. (uredinia and telia). 


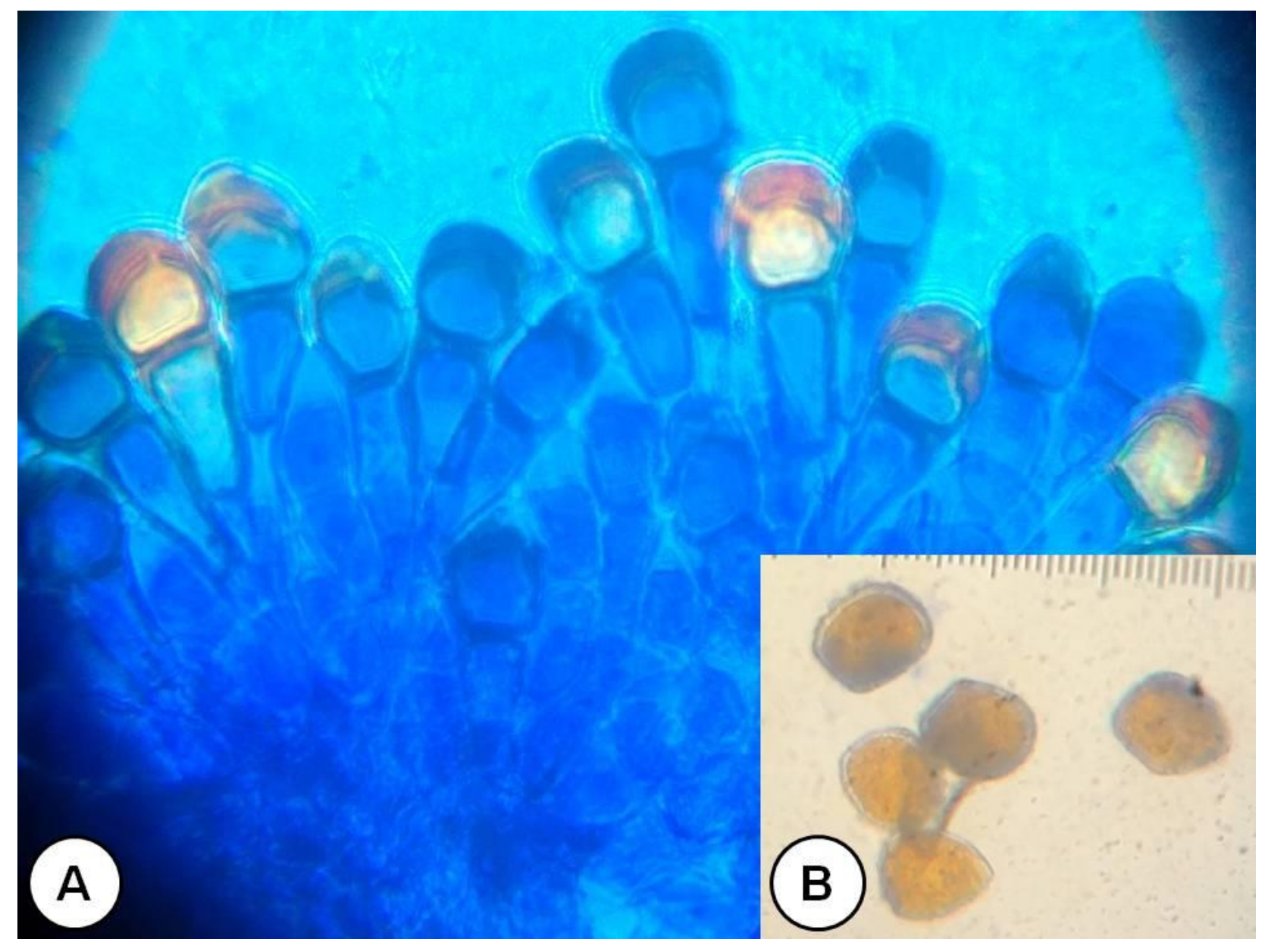

Fig 3 - Microscopic characteristics of Puccinia. A teliospores; B urediniospores.

A complete list of Puccinia species recorded in Himachal Pradesh, India is presented in alphabetical order followed by family of host plant and related reference. Apart from the distribution in Himachal Pradesh, the distribution of Puccinia outside the state is also provided.

Order: Puccinales, Family: Puccinaceae, Genus: Puccinia

Puccinia agrostidis Plowr. (Ranunculaceae) (Barclay 1891)

Distribution: Shimla (H.P.).

Puccinia ahmadiana Syd. (Asteraceae) (Sydow 1938)

Distribution: Reported from various localities including Puti Ruhi, Lahul, Kullu Valley of Himachal Pradesh. No reports are available from outside the state.

Puccinia allii (DC.) F. Rud. (Amaryllidaceae) (Butler \& Bisby 1931)

Distribution: Kullu (H.P.).

Puccinia andropogonis Schw. (Poaceae) (Barclay 1890 a, b)

Distribution: Shimla (H.P.).

Puccinia anthistiriae Barclay (Poaceae) (Sydow \& Butler 1912)

Distribution: Reported form Shimla (H.P.). It is also reported from Harwan (J\&K). 
Puccinia apii Desm. (Apiaceae) (Barclay 1890 a, b)

Distribution: Shimla (H.P.).

Puccinia arenariae (Schumacher) J. Schröt. (Caryophyllaceae) (Barclay 1891)

Distribution: Narkanda Shimla (H.P.).

Puccinia arthraxonis (P. Henn.) Syd., P. Syd. \& E.J. Butler (Poaceae) (Golatkar 1976)

Distribution: Reported from Kasauli (H.P.) and from Dehradoon, Kumaon and Massoori (Uttk.).

Puccinia atropuncta Peck \& Clint. (Asteraceae) (Chona et al. 1956)

Distribution: Shimla (H.P.).

Puccinia bulbocastani (A. Cumino) Fuckel (Apiaceae) (Bhardwaj \& Sharma 1990)

Distribution: Reported only from Himachal Pradesh.

Puccinia bistortae (F. Strauss.) DC. (Polygonaceae) (Sydow 1938)

Distribution: Losar, Spiti (H.P.).

Puccinia brachypodii G.H. Otth. (Berberidaceae) (Payak 1965)

Distribution: Shimla (H.P.).

Puccinia bupleuri-falcate (DC.) G. Wint. (Apiaceae) (Barclay 1890 a, b)

Distribution: Reported from Shimla (H.P.). It was also reported from Rajendragarh $(\mathrm{Pb})$ and Nainital (Uttrk.).

Puccinia calthae Link (Ranunculaceae and Berberidaceae) (Arthur \& Cummins 1933, Chona \& Munjal et al. 1956)

Distribution: In Himachal Pradesh the rust was reported from Dhramshala; Rohtang pass, Kullu, while Pahalgam from (J\&K).

Puccinia caricis var. himalayensis Barclay (Cyperaceae) (Butler \& Bisby 1931, Padwick \& Khan 1944)

Distribution: Reported from Shimla (H.P.) and Mussoorie (Uttk.).

Puccinia caricis-filicinae Barclay (Cyperaceae) (Mitter \& Tandon 1938)

Distribution: Reported from Shimla (H.P.) and Ranikhet \& Nainital (Uttk.).

Puccinia caricis-nubigenae Padwick \& A. Khan (Cyperaceae) (Mitter \& Tandon 1938)

Distribution: Reported only from Kufri, Shimla (H.P.).

Puccinia carthami Corda (Asteraceae) (Sydow \& Butler 1901)

Distribution: Reported from Kangra (H.P.) and Bihar, Punjab, Hydrabad (A.P.).

Puccinia chrysopogoni Barclay (Oleaceae and Poaceae) (Barclay 1890 a, b, Sydow \& Butler 1907) Distribution: Reported from Shimla (H.P.), Kumaon \& Jaunsar (Uttk) and Aurangabaad (M.S.).

Puccinia circaeae Pers. (Onagraceae) (Barclay 1890 a, b)

Distribution: Reported from Shimla (H.P.), Aru (J\&K) and Nainital (Uttk).

Puccinia collettiana Barclay (Rubiaceae) (Barclay 1890 a, b, Ganguly \& Pandotra 1963)

Distribution: Reported from Shimla, Kasauli, Nagar (Kullu) of Himachal Pradesh, Ranikhet, Mussoorie \& Nainital (Uttk) and Mahabaleshwar (M.S.). 
Puccinia coronata var. avenae P. Syd. \& Syd. (Poaceae) (Mishra et al. 1964)

Distribution: Reported from Shimla (H.P.) while Kalimpong \& Darjling from West Bengal.

Puccinia coursiniae P. Syd. \& Syd. (Poaceae) (Padwick 1945)

Distribution: Spiti (H.P.).

Puccinia cynodontis Lacroix ex Desm. (Poaceae) (Sharma \& Sachan 1994)

Distribution: In addition to H.P., the fungus is also reported from Saharanpur (U.P.); Poona (M.S.);

Kanpur (U.P.); Surat (GJ); Kashmir (J\&K); Muzaffarpur (Bihar); Gwalior \& Jabalpur (M.P.).

Puccinia dactylidina Bubak (Poaceae) (Sydow \& Butler 1912)

Distribution: Reported from Shimla (H.P.) and Harwan (J\&K).

Puccinia dioscoreae Kom (Dioscoreaceae) (Pandotra \& Ganguly 1962)

Distribution: Manali (H.P.).

Puccinia duthiae Ellis \& Tracy (Poaceae) (Sydow \& Butler 1911)

Distribution: This fungus has a very wide distribution. In Himachal Pradesh it was reported from Kasauli and Dehradoon (Uttk.), Poona (M.S.), Phulguru, Jabalpur (M.P.), Dharwar (Kanataka), and Rajgir (Bihar)

Puccinia ellisii De Toni (Apiaceae) (Barclay 1891)

Distribution: Reported only from Phagu, Shimla (H.P.).

Puccinia eremuri Kom. (Xanthorrhoeaceae) (Barclay 1891)

Distribution: Kullu (H.P.).

Puccinia erianthi Padwick \& A. Khan (Poaceae) (Padwick \& Khan 1944)

Distribution: The fungus was reported from Shimla (H.P.) and from Coimbatore (T.N.), Hydrabeead (A.P.), New Delhi.

Puccinia eulaliae Barclay (Poaceae) (Butler \& Bisby 1960)

Distribution: Reported from Shimla (H.P.) only.

Puccinia excelsa Barclay (Lamiaceae) (Barclay 1891)

Distribution: Reported from Mahasu \& Huttoo Peak, Shimla (H.P.).

Puccinia fagopyri Barclay (Polygonaceae) (Barclay 1890 a, b)

Distribution: Reported form Shimla \& Sangla valley (H.P.) and Mussoorie (Uttrakhand).

Puccinia flavipes Syd. \& P. Syd. (Rosaceae) (Barclay 1890 a, b)

Distribution: Shimla (H.P.).

Puccinia gentianae (F. Strauss.) Link. (Gentianaceae) (Barclay 1890 a, b)

Distribution: Reported from Shimla (H.P.) and Chillam (North West Himalayan region including Uttk.).

Puccinia geranii-silvatici P. Karst. (Geraniaceae) (Barclay 1890 a, b)

Distribution: Reported from Shimla (H.P.) and Verinag (J\&K). 
Puccinia glumarum (Schw.) Eriks \& P. Henn. (Poaceae) (Prasada 1948)

Distribution: In Himachal Pradesh the fungus was reported from Shimla while from Hisar (HR), Dehradoon (Uttk.) outside the state.

Puccinia graminis Pers. (Poaceae) (Barclay 1890a)

Distribution: Reported from entire states of Northern India including Shimla, Kullu (H.P.) and Harwan (J\&K), Nainital (Uttrakhand), New Delhi, Hsar (Haryana) and even from Kolhapur (Maharastra), Jabalpur (M.P.).

Puccinia graminis-agropyri P.R. Mehta \& R. Prasad (Poaceae) (Prasada 1948)

Distribution: Shimla (H.P.).

Puccinia graminis-poae (Pers.) Erikss. \& Henning (Poaceae) (Prasada 1948)

Distribution: Shimla (H.P.).

Puccinia himalensis (Barclay) Diet. (Rhamnaceae, Poaceae) (Padwick 1946, Sydow \& Butler 1906 \& 1907)

Distribution: Reported from Shimla (H.P.) and Kuaon, Nainital (Uttk.) and Harwan (J\&K).

Puccinia invenusta Syd. \& P. Syd. (Poaceae) (Sharma \& Sachan 1994)

Distribution: In addition to H.P., also reported from Bihar, Delhi, Assam and U.P.

Puccinia iridis (DC.) Wallr. (Iridaceae) (Sydow \& Butler 1912)

Distribution: Reported from Shimla in the state of Himachal Pradesh. Outside the state it was reported from Harwan, Srinagar, Jammu (J\&K), Mussoorie (Uttk.) and Kohima (NL).

Puccinia komarovi Tranzschel ex P. Syd. \& Syd. (Balsaminaceae) (Khanna 1961)

Distribution: Reported from Shimla (H.P.) and Mussoorie (Uttk.) and Sonamarg (J\&K).

Puccinia leptodermidis Barclay (Rubiaceae) (Barclay 1890 a, b, Sydow \& Butler 1912)

Distribution: Shimla \& Kasauli (H.P.).

Puccinia lycoctoni Fuckel (Ranunculaceae) (Sydow \& Butler 1907)

Distribution: Shimla (H.P.).

Puccinia menthae Pers. (Lamiaceae) (Pandotra \& Ganguly 1964, Sydow \& Butler 1912, Sydow 1938)

Distribution: This species has a wide host distribution. It was reported from Shimla and Kasauli, Kullu, Busher in Himachal Pradesh, whereas, from Harwan \& Verinag, Shadipur, Landhari, Batote in J\&K, Mussoorie, Ranikhet in Uttrakhand, Bilaspur (M.P.) and Kohima (NL).

Puccinia minutissima Arthur (Poaceae) (Munjal \& Gill 1962)

Distribution: Reported from Manali (H.P.) and Mussoorie (Uttk.).

Puccinia nepalensis Barclay \& Dietel (Polygonaceae) (Barclay 1890 a, b, Ramakrishanan 1952) Distribution: Reported from Shimla and Kasauli (H.P.), Mussoorie \& Nainital (Uttk.), Ootacamund (TN) and Harwan (J\&K).

Puccinia neyaudiae Syd. (Poaceae) (Sharma \& Sachan 1994)

Distribution: The fungus was also reported from Darjeeling (W.B.) in addition to H.P. 
Puccinia nitida (F. Strauss) Barclay (Polygonaceae) (Sydow \& Butler 1912)

Distribution: Reported from Mashobra, Shimla, Rohtaang Pass (H.P.) and Tukwar, Darjling (W.B.), Mussoorie \& Nainital (Uttk.), Jammu, Verinag and Banihal Pass (J\&K).

Puccinia opizii Bubák (Asteraceae) (Arthur \& Cunmins 1933)

Distribution: Reported only from Alwas (Chamba) of Himachal Pradesh.

Puccinia pacifica Blasdale ex Arthur (Plantaginaceae) (Chona et al. 1956)

Distribution: Shimla (H.P.).

Puccinia persistens Plowr. (Ranunculaceae) (Barclay 1890 a, b)

Distribution: Reported from Shimla (H.P.) and Nainital (Uttk.).

Puccinia pimpinellae (F. Strauss) Roehling (Apiaceae) (Barclay 1890 a, b)

Distribution: Reported from Shimla (H.P.), Kumaon \& Mussoorie (Uttk.) Harwan \& Batote (J\&K).

Puccinia polliniae Barclay (Acanthaceae) (Barclay 1890 a, b)

Distribution: Reported from Shimla (H.P.), Nainital, Dehradoon \& Mussoorie (Uttk.) and Mysore (Karnataka).

Puccinia pogonatheri Petch (Poaceae) (Sharma \& Sachan 1994)

Distribution: In addition to Himachal Pradesh, also reported from Singampath (T.N.).

Puccinia polygoni Alb. \& Schw. (Polygonaceae) (Ganguly \& Pandotra 1963)

Distribution: Reported from Katrain (H.P.) and Mussoorie, Nainital (Uttk.) and Jammu (J\&K).

Puccinia polygoni-amphibii Pers. (Polygonaceae) (Mishra \& Sharma 1964)

Distribution: Reported from Shimla (H.P.) and also from Mussoorie (Uttk.) and Harwan, Srinagar (J\&K), Jabarkhet Landour (U.P.) and Assam.

Puccinia prainiana Barclay (Smilacaceae) (Barclay 1890 a, b)

Distribution: This fungus has a very wide distribution in India. In Himachal Pradesh it was reported form Shimla. In rest of the country reported from Mussoorie, Ranijhet \& Kumaon (Uttk.), Droog, Nilgiris (T.N.), Wynaad (Kerela), Khasi Hills (Assam), Yercaud (Karnataka), Purnea (Bihar), and Mahableshwar, Kanheri forests, Amboli Panhala (MS)

Puccinia punctata Link (Rubiaceae) (Barclay 1890 a, b)

Distribution: Reported from Shimla (H.P.), Nainital (Uttk.) and Batote (J\&K).

Puccinia purpurea Cooke (Poaceae) (Sharma \& Sachan 1994)

Distribution: In addition to Himachal Pradesh also reported from Dehradoon, (Uttk.); Patna (Bihar); Arvu \& Phulgaon, Jabalpur (M.P.); Mount Abu (Raj); Coimbatore (T.N.), Poona, Kolhapur (M.S.); Surat (GJ.); Ranchi (Chattisgarh); Dharwar (Karnataka).

Puccinia pusilla Syd. \& P. Syd. (Poaceae) (Sharma \& Sachan 1994)

Distribution: In addition to Himachal Pradesh the fungus was also reported from Dehradoon, Ranikhet, Bhowali (Uttk.); Parasnath Hills (Bihar); Jabalpur (M.P.); Assam and Idukki (Kerela).

Puccinia recondita var. simlensis Misra, Ahmad \& Singh. (Ranunculaceae and Poaceae) (Gupta 1977)

Distribution: Shimla (H.P.). 
Puccinia roscoeae Barclay (Zingiberaceae) (Gupta 1977)

Distribution: Reported from Shimla (H.P.) and Mussoorie (Uttk.).

Puccinia rubigo-vera (DC.) G. Winter (Ranunculaceae) (Arthur \& Cummins 1933, Jain et al. 1966)

Distribution: Reported form Dharamshala, Chamba, Kote, Keylog, Kullu in Himachal Pradesh. It was also reported from Sonamarg, Kashmir (J\&K) and Kanpur (U.P.)

Puccinia saviculae Grev. (Apiaceae) (Barclay 1890 a, b)

Distribution: Reported from Shimla (H.P.) and Kumaon (Uttk.).

Puccinia saxifragae-cilliatae Barclay (Saxifragaceae) (Barclay 1890 a, b)

Distribution: Reported from Shimla (H.P.), Mussoorie \& Nainital (Uttk.) Jehlam valley, Kilanmarg $(\mathrm{J} \& \mathrm{~K})$.

Puccinia saxifragae-micranthae Barclay (Saxifragaceae) (Barclay 1891)

Distribution: Bushar \& Shimla (H.P.).

Puccinia sonchi Roberge ex Desm. (Asteraceae) (Sydow 1938)

Distribution: Reported from Kullu (H.P.) and Koppa, Mysore (Karnataka), Srinagar (J\&K) and Kumaon (Uttk.).

Puccinia sorghi Schwein. (Poaceae and Oxalidaceae) (Annonymous 1950, Mishra 1963)

Distribution: Reported from Mashobra \& Shimla (H.P.). It was also reported from Manjiri, Mumbai (MS.), Kashmir (J\&K), Dumraon (Bihar).

Puccinia striiformis Westend. (Poaceae) (Vasudeva 1958, Joshi \& Merchand 1963, Mishra et al. 1965, 1975, Ahmad et al. 1969)

Distribution: In Himachal Pradesh the fungus was reported only from Shimla but also from Punjab, Jabalpur (M.P.) and Jhansi (U.P.) outside the state.

Puccinia striiformis f. muehlenbergii Misra \& Lele. (Poaceae) (Mishra \& Lele 1963)

Distribution: Reported only from Shimla (HP).

Puccinia tanaceti DC. (Asteraceae) (Sharma \& Sachan 1994, Bharat 2008)

Distribution: In addition to Himachal Pradesh, also reported from Kashmir (J\&K) and Bihar.

Puccinia thlaspeos Ficinus \& C. Schub. (Arthur 1934, Arthur \& Cummins 1933) Brassicaceae Distribution: The fungus was reported only from Hunan Nallah, Pangi \& Chamba (H.P.).

Puccinia tricholepidis Syd. (Asteraceae) (Sydow 1938)

Distribution: Saharan \& Bushar (H.P.).

Puccinia trolii P. Karst. (Ranunculaceae) (Arthur \& Cummins 1933)

Distribution: Reported only from Alwas, Chamba (H.P.).

Puccinia tweediana (Speg.) T.S. Ramakr. \& K. Ramakr. (Acanthaceae) (Chona \& Munjal 1955, Ramakrishnan \& Ramakrishnan 1948)

Distribution: Reported from Kullu; Shimla in Himachal Pradesh whereas, from Yercaud (T.N.) and Mussoorie (Uttk.). 
Puccinia urticae Barclay (Urticaceae) (Barclay 1890 a, b)

Distribution: Reported from Shimla \& Kasauli (H.P.) and Mussoorie \& Nainital (Uttk.).

Puccinia ustalis Berk. (Ranunculaceae) (Berkeley 1856)

Distribution: Mathana, Shimla (H.P.).

Puccinia violae (Schumach.) DC. (Violaceae) (Bilgrami 1963)

Distribution: Reported from Shimla (H.P.), Harwan (J\&K) and Nainital \& Mussoorie (Uttk.).

\section{Host index}

In this section scientific names of host plants along with the Puccinia species occurring on them are provided.

Aconitum lycoctonum - Puccinia lycoctoni.

Agropyron semicostatum - Puccinia graminis-agropyri

Agrosotis stolonifera - Puccinia himalensis

Allium sativum - Puccinia allii

Andropogon tristis - Puccinia andropogonis

Andropogon pertusus - Puccinia duthiae

Anemone polyanthes - Puccinia trolii

Angelica glauca - Puccinia ellisii

Anthistiria anathera - Puccinia anthistiriae

Apium graveolens - Puccinia apii

Aquilegia vulgaris - Puccinia agrostidis

Aquilegia vulgaris - Puccinia rubigo-vera

Artemisia nilogirca - Puccinia tanaceti

Arthraxon prionodes - Puccinia arthraxonis

Avena sativa - Puccinia coronata var. avenae

Berberis aristata - Puccinia brachypodii

Berberis lyceum - Puccinia brachypodii

Brachypodium sylvaticum - Puccinia glumarum

Brchaypodium sylvaticum - Puccinia himalensis

Bromus japonicas - Puccinia striiformis

Bunium persicum - Puccinia bulbocastani

Bupleurum falcatum - Puccinia bupleuri-falcate

Caltha palustris var. alba-Puccinia calthae

Caltha palustris var. normalis - Puccinia calthae

Capillipendium assimile - Puccinia pusilla

Carex filicina - Puccinia caricis-filicinae

Carex nubigena - Puccinia caricis-nubigenae

Carex setigera-Puccinia caricis var. himalayensis

Carthamus oxycantha - Puccinia carthami

Chrysopogon gryllus - Puccinia chrysopogi

Circaea alpine - Puccinia circaeae

Clematis gouriana - Puccinia walliana

Coursinia thomsoni-Puccinia coursiniae

Cynodon dactylon - Puccinia cynodontis

Dactylis glomerata - Puccinia dactylidina

Dicliptera bupleuroides - Puccinia tweediana

Dicliptera sp. - Puccinia tweediana

Dioscorea deltoidea - Puccinia dioscoreae

Draba laneolata - Puccinia thlaspeos

Eremurus himalaicus - Puccinia eremuri 
Erianthus fulvus - Puccinia erianthi

Fagopyrum esculentum - Puccinia fagopyri

Festuca gigentia - Puccinia graminis

Festuea gigentia - Puccinia himalensis

Fragaria vesca-Puccinia flavipes

Galium aparnie - Puccinia puctata

Gentiana kurroo - Puccinia gentianae

Geranium nepalense - Puccinia geranii-sylvaticae

Rhamnus dahurica - Puccinia himalensis

Thalictrum javanicum - Puccinia recondita var. simlensis

Helicotrichon virens - Puccinia recondita var. simlensis

Impatiens amphorata - Puccinia komarovi

Iris florentina - Puccinia iridis

Iris pallid-Puccinia iridis

Jasminum humile - Puccinia chrysopogi

Lactuca decipiens - Puccinia opizii

Leptodermis lenceolata - Puccinia leptodermidis

Lolium perenne - Puccinia striiformis

Mentha longifolia - Puccinia menthae

Mentha sylvestris - Puccinia menthae

Muehlenbergia huegelii-Puccinia striiformis

Neyaudia arundi-aceaea - Puccinia neyaudiae

Origanum vulgare - Puccinia menthae

Oryzopsis holiciforme - Puccinia himalensis

Oxalis corniculata - Puccinia sorghi

Oxalis latifolia - Puccinia sorghi

Phlomis bracteosa - Puccinia excels

Phramites karka-Puccinia invenusta

Pimpinella diversifolia - Puccinia pimpinellae

Plantago tibetica - Puccinia pacifica

Poa nemoralis - Puccinia graminis-poae

Pogonatherum paniceum - Puccinia pogonatheri

Pollinia japonica - Puccinia eulaliae

Pollinia nuda - Puccinia polliniae

Polygonum ampelexicaule - Puccinia nitida

Polygonum orintale - Puccinia polygone

Polygonum viviparum - Puccinia bistortae

Pogonatherum paniceum - Puccinia pogonatheri

Prenanthes brunoniana - Puccinia atropuncta

Pterotheca falconeri - Puccinia ahmadiana

Ranunuculus hirtellus - Puccinia ustalis

Rhamnus dahurica - Puccinia himalensis

Rhamnus virgeta - Puccinia himalensis

Roscoea alpine - Puccinia roscoeae

Roscoea procera - Puccinia roscoeae

Rubia cordifolia - Puccinia colletiana

Rumex nepalensis - Puccinia nepalensis

Saccharum officinarum - Puccinia minutissima

Savicula europea - Puccinia saviculae

Saxifraga ligulata var. cilliata-Puccinia saxifragae-cilliatae

Saxifraga micrantha-Puccinia saxifragae-micranthae

Smilax aspera - Puccinia prainiana 
Sonchus sp. - Puccinia sonchi

Sorghum halepense - Puccinia purpurea

Stellaria paniculata - Puccinia arenariae

Strobilanthes dalhausianus - Puccinia polliniae

Thalictrum javanicum - Puccinia persistens

Thalictrum javanicum - Puccinia recondita var. simlensis

Thalictrum minus - Puccinia rubigo-vera

Thalictrum sp. - Puccinia rubigo-vera

Tricholepis elongate - Puccinia tricholepidis

Triticum vulgare - Puccinia striiformis

Urtica parviflora - Puccinia caricis var. himalayensis

Urtica parvilflora - Puccinia urticae

Viola serpens - Puccinia violae

Zea mays - Puccinia sorghi

\section{Discussion}

The genus Puccinia is the largest in the Pucciniales and is considered as the most economically important genus of biotropic fungi. Members of this genus are ubiquitous plant pathogens and affect almost all types of plants. A very broad host range of the genus makes it responsible for numerous plant diseases having very serious consequences.

It is observed that almost all the reports are from Shimla and nearby regions. So other regions of the state should also be explored in order to explore the biodiversity and host range of the fungi. Additionally, we did not find any information on molecular identification of Puccinia species from the state. Therefore, more studies including molecular work are required in order to confirm the fungal species.

\section{Acknowledgements}

Authors thank their respective organizations for providing every possible help to complete this work successfully.

\section{References}

Ahmad ST, Singh HS, Mishra DP. 1969 - Additions to wheat rusts races in India race $14 \& 38$ of Puccinia striformis identified during 1965. Indian Phytopathology 22, 525-526.

Annonymous. 1950 - List of common names of Indian plant diseases. Indian Journal of Agricultural Research 20, 107-142.

Arthur JC. 1934 - Manual of rusts in United States and Canada. Lafayette, Indiana.

Arthur JC, Cummins GB. 1933 - Rusts of North West Himalayas. Mycologia 25, 397-406.

Barclay A. 1890a - Descriptive list of Uredineae occurring in the neighborhood of Shimla. Journal Asiatic Society of Bangal 56, 161-165.

Barclay A. 1890b - Descriptive list of Uredineae occurring in the neighborhood of Shimla. Journal Asiatic Society of Bangal 56, 350-375.

Barclay A. 1891 - Additional Uredineae from the neighborhood of Shimla. Journal Asiatic Society of Bangal 60, 211-230.

Berkeley MJ. 1856 - Decades of fungi; Decas 1-62 nos 1-620. Hooker's London Journal of Botany 3-8, 844-1856.

Bhardwaj LN, Sharma PN. 1990 - Occurrence of rust on black zira in Himachal Pradesh. Indian Journal of Mycology and Plant Pathology 20 (3), 270.

Bilgrami KS. 1963 - A new species of Periconia on the leaves of Typha latifolia. Science and Culture 29, 47-48.

Bharat NK. 2008 - Occurrence of rust on Artemisia dracunculus L. in Himachal Pradesh. Indian Forester 134, 140. 
Butler EJ, Bisby GR. 1931 - The fungi of India. Imperial Council of Agricultural Research India. Science Monograph I XVIII+237pp.

Butler EJ, Bisby GR. (Revised by Vasudeva RS.) 1960 - The fungi of India. ICAR Publications New Delhi, India, 552 pp.

Chona BL, Munjal RL. 1955 - Notes on miscellaneous fungi - II. Indian Phytopathology 8, 184198.

Chona BL, Munjal RL. 1956 - Notes on miscellaneous fungi-III. Indian Phytopathology 9, 53-56.

Chona BL, Munjal RL, Kapoor JN. 1956 - Notes on miscellaneous Indian fungi-IV. Indian Phytopathology 9, 125-132.

Ganguly D, Pandotra VR. 1963 - Fungi on medicinal and aromatic plants in North West Himalays - I. Mycopathologia et Mycologia Applicata 20, 39-40.

Golatkar VV. 1976 - Harknessia: a new generic record to India. Current Science 45, 269-270.

Gupta SC. 1977 - New diseases of sugarcane in U.P. India. Current Science 46, 27.

Jain AC, Nikam BG, Kulkarni SN, Sharma OP. 1966 - Fungi of Gwalior and Indore region. The Vikram 4, 181-187.

Joshi LM, Merchand WC. 1963 - Bromus japonicas Thunb. susceptible to wheat rust under normal conditions. Indian Phytopathology 16, 312-313.

Khanna PK. 1961 - A new report of a new host of Puccinia kanmorensis Cummins from India. Proceedings from National Academy of Sciences India. $113 \mathrm{pp}$.

Mishra DP. 1963 - Natural occurrence of the aecial stage of Puccinia sorghi Schw. Oxalis corniculata Linn. in Nepal. Indian Phytopathology 16, 8-9.

Mishra DP, Lele VC. 1963 - Choanephora cucubitarum on Cajanus cajan in India. Indian Phytopathology 22, 515-517.

Mishra DP, Sharma SK. 1964 - Natural infection of Oxalis corniculata, the alternate host of Puccinia sorghi in India. Indian Phytopathology 17, 138-141.

Mishra DP, Sharma SK, Joshi PC, Singh S. 1964 - Further studies in the crown rust of oat in India. Indian Phytopathology 17, 142-145.

Mishra DP, Ahmad ST, Singh HS. 1965 - Natural occurrence of specialized forms of Puccinia graminis and Puccinia striiformis on Lolium perenne. Indian Phytopathology 18, 214-215.

Mishra DP, Singh S, Ahmad S. 1975 - Notes on some rusts from Shimla Hills. Indian Phytopathology 28, 256-260.

Mitter JH, Tandon RN. 1938 - Fungi of Nainital Part-II. Journal of Indian Botanical Society 17, $177-182$.

Munjal RL, Gill HS. 1962 - Some dematiaceous fungi from India-I. Indian Phytopathology 15, 269-279.

Padwick GW. 1945 - Notes on Indian fungi-III. Mycological Papers 12, 15 pp.

Padwick GW. 1946 - Notes on Indian fungi-IV. Mycological Papers 17, 12 pp.

Padwick GW, Khan A. 1944. Notes on Indian fungi-II. Mycological Papers 10, 17 pp.

Pandotra VR, Ganguly D. 1962 - Notes on two fungi collected from Kullu Valley, Punjab. Indian Phytopathology 15, 216-217.

Pandotra VR, Ganguly D.1964 - Fungi on medicinal and aromatic plants in North West Himalayas I. Mycopathology et Mycological Applicata 22, 59-68.

Payak MM. 1965 - Berberis as the aecial host of Puccinia brachypodii in Shimla Hills, India. Phytopathology 52, 49-54.

Prasada R. 1948 - Studies on rusts on some of the wild grasses occurring in the neighborhood of Shimla. Indian Journal of Agricultural Science 18, 165-176.

Ramakrishanan TS. 1952 - Additions to fungi of Madras-XII. Proceeings of Indian Academy of Sciences 35(B), 11-121.

Ramakrishnan TS, Ramakrishanan K. 1948 - Additions to fungi of Madras- V. Proceedings of Indian Academy of Sciences 28(B), 50-70.

Sharma RK, Sachan SN. 1994 - New host of rust fungi from Himachal Pradesh. Advances in Plant Sciences 7, 154-158. 
Sydow H. 1938 - Fungi Himalayensis. Annals of Mycology 36, 437-442.

Sydow H, Butler EJ. 1901 - Fungi Indiae Orientalis Pars. I. Annals of Mycology 4, 424-445.

Sydow H, Butler EJ. 1906 - Fungi Indiae Orientalis Pars. I. Annales Mycologici 4, 424-445.

Sydow H, Butler EJ. 1907 - Fungi Indiae Orientalis II. Annales Mycologici 5, 485-515.

Sydow H, Butler EJ. 1911 - Fungi Indiae Orientalis part III. Annales Mycologici 9, 372-421.

Sydow H, Butler EJ. 1912 - Fungi Indiae Orientalis IV. Annales Mycologici 10, 243-280.

Vasudeva RS. 1958 - Report of the division of Mycology and Plant Pathology. Science Report of Agricultural Research Institute, New Delhi. 87-101 \& 1954-55. 